\title{
Remote Ultra-Low Light Imaging (RULLI) for Space Situational Awareness (SSA): Modeling and Simulation Results for Passive and Active SSA
}

\author{
Michael C. Roggemann ${ }^{1}$, Kris Hamada ${ }^{2}$, S. Rao Gudimetla ${ }^{3}$, \\ Kim Luu ${ }^{3}$, L. William Bradford ${ }^{2}$, David C. Thompson ${ }^{4}$, Robert Shirey ${ }^{4}$
}

\begin{abstract}
Remote Ultra-Low Light Imaging detectors are photon limited detectors developed at Los Alamos National Laboratories. RULLI detectors provide a very high degree of temporal resolution for the arrival times of detected photoevents, but saturate at a photo-detection rate of about $10^{6}$ photo-events per second. Rather than recording a conventional image, such as output by a charged coupled device (CCD) camera, the RULLI detector outputs a data stream consisting of the two-dimensional location, and time of arrival of each detected photo-electron. Hence, there is no need to select a specific exposure time to accumulate photo-events prior to the data collection with a RULLI detector - this quantity can be optimized in post processing. RULLI detectors have lower peak quantum efficiency (from as low as $5 \%$ to perhaps as much as $40 \%$ with modern photocathode technology) than back-illuminated CCD's ( $80 \%$ or higher). As a result of these factors, and the associated analyses of signal and noise, we have found that RULLI detectors can play two key new roles in SSA: passive imaging of exceedingly dim objects, and three-dimensional imaging of objects illuminated with an appropriate pulsed laser. In this paper we describe the RULLI detection model, compare it to a conventional CCD detection model, and present analytic and simulation results to show the limits of performance of RULLI detectors used for SSA applications at AMOS field site.
\end{abstract}

Keywords: atmospheric optics, imaging through turbulence

\section{INTRODUCTION}

Remote Ultra-Low Light Imaging (RULLI) detectors have been developed at Los Alamos National Laboratories (LANL) for the purposes of two dimensional (2-D) passive imaging and three-dimensional (3-D) active imaging at visible wavelengths [Priedhorsky, 1996a; Priedhorsky, 1996b; Ho, 1999; Priedhorsky, 2005]. The RULLI detector combines photon-limited detection with a precise timing capability. Physically, a RULLI detector consists of photocathode, followed by a micro channel plate gain stage. The electron cloud generated by the gain stage falls on a crosseddelay line detection system, and the electronics associated with this readout mechanism account for both the photonlimited nature of the detection, and the high degree of temporal resolution. The data stream output by the RULLI detector is a sequence of photo-electron locations and arrival times. The RULLI detector has nether pixels nor frames; the data stream output by the RULLI detector is a sequence of photo-electron locations and arrival times. Both position and time data have very high resolution, but electron optics and other effects give rise to point spread functions in space and time with widths equivalent to $\sim 100 \mathrm{um}$ for a $40 \mathrm{~mm}$ diameter detector and an absolute time resolution of $\sim 100 \mathrm{ps}$. The RULLI detector also has extremely low dark current, but has a relatively low saturation count rate of about $10^{6}$ photo-electrons/second. These properties of the RULLI detector make it a favorable option for both passive 2-D imaging of very dim objects, and 3-D imaging using pulsed laser illumination of targets.

In this paper we examine the roles of a RULLI detector in passive and active imaging for space situational awareness (SSA) applications. We have developed a simulation of RULLI performance for speckle imaging through atmospheric turbulence [Roggemann, 1996], and done a comparison of imaging performance with a RULLI detector and a conventional CCD detector using signal-to-noise ratio (SNR) as the comparison metric. We have also developed a simulation of 3-D imaging with the RULLI sensor based on a short-pulse laser illuminator operating at $532 \mathrm{~nm}$. Here we present the models, and some representative results. We find that the RULLI detector will provide better SNR than a

\footnotetext{
${ }^{1}$ Michigan Technological University, Houghton, MI, and Pacific Defense Solutions, Kihei, HI

${ }^{2}$ Pacific Defense Solutions, Kihei, HI

${ }^{3}$ Air Force Research Laboratory, Maui Space Surveillance Site, Kihei, HI

${ }^{4}$ Los Alamos National Laboratory, Los Alamos, NM 87545
} 
CCD detector for very dim objects. In the case of 3-D imaging we modeled a relatively small transmitter aperture with a diverging beam providing illumination, and an adaptive optics-equipped telescope with a RULLI detector as the receiver. In this scenario resolution in the plane transverse to the direction of the illuminating beam is associated with the adaptive optics, while resolution along the direction of the illuminating beam, that is, in range, is due to the combination of the use of a pulsed laser and the RULLI detection system. In the case of 3-D imaging we find that excellent 3-D visualizations of the target are feasible under realistic seeing and illumination conditions.

The remainder of this paper is organized as follows. In the next section we discuss the RULLI detector model. We then describe the 2-D and 3-D simulations. Representative results are provided within the discussions of the 2-D and 3-D models. Conclusions are presented in the final section of the paper.

\section{1.}

The RULLI detector can be reasonably modeled as a photon-limited, time resolved detector. Our model of the RULLI detector function is based on the published literature [Priedhorsky, 1996; Priedhorsky, 2005; Ho, 1999]. After calibration, the data available from the RULLI is a matrix with $K$ rows, where $K$ is the total number of photo-electrons collected, and 3 columns, one each for the coordinates of the $k^{t h}$ photo-event, $\left(x_{k}, y_{k}\right)$, and one for the arrival time $t_{k}$, where the subscript $k$ is an integer, $1 \leq k \leq K$. Under reasonable assumptions about the conditions of pulsed laser illumination the objects can be assumed to be spatially incoherent, and hence, the images formed by the optical system are incoherent images [Goodman, 1996], which have a narrow spectral bandwidth. We represent the classical intensity falling on the detector at time $t$ as $i(x, y ; t)$, where $(x, y)$ represents an image plane coordinate. To model a RULLI detector it is necessary to establish within the simulation a temporal resolution which we represent by $\Delta t$. The smallest value that $\Delta t$ can take is the temporal resolution of the RULLI, which is on the order of $100 \mathrm{ps.} \mathrm{However,} \mathrm{we} \mathrm{generally}$ run the simulation at larger values of $\Delta t$ for the following reasons:

1. In the case of 2-D imaging it is necessary to integrate the images for a finite amount of time to collect photoevents in a 2-D image before the images are post processed. For imaging space objects through atmospheric turbulence, the integration time is generally limited by the temporal dynamics of the atmospheric turbulence to be in the range of a few ms to a few tens of ms.

2. In the case of 3-D imaging the temporal resolution setting establishes the resolution in the direction of propagation of the illuminating beam, which we shall henceforth refer to as the $z$ direction. The resolution in the $z$ direction is given by $\Delta z=c \Delta t / 2$, where $c$ is the speed of light. For the case of the RULLI running at its maximum temporal resolution $\Delta t=100 \mathrm{ps}$ this corresponds to range resolution of $\Delta z=15 \mathrm{~mm}$. Setting $\Delta z$ this small gives rise to two practical problems: (a) In the simulation it is necessary to "slice" the target in the $z$ direction into a number of slabs which is approximated by $D / \Delta z$, where $D$ is the depth of the target in the $z$ direction. For reasonably sized targets, $D \approx 10 \mathrm{~m}$, so that the number of slabs required is on the order of 660. The targets must also be sampled in image space at the Nyquist rate for the aperture (i.e., an angular sampling rate of $\Delta \alpha=\lambda /\left(2 D_{R}\right)$, where $\lambda$ is the mean wavelength of operation and $D_{R}$ is the receiver pupil diameter), often leading to array sizes on the order of $256 \times 256$ samples, or more, to hold the target. Hence, holding all the this target data in memory requires a $256 \times 256 \times 660$ sample array, which can easily exceed the memory capabilities of PC computers. Additionally, the target models available for this study have very little detail on the scale of $15 \mathrm{~mm}$.

As a result of these considerations, the temporal resolution of the RULLI was set in the following manner for the 2-D imaging simulations. The mean photo-electron generation rate $\bar{K}$, with units of counts per second, is set by the user. $\bar{K}$ could be calculated from radiometric considerations for very dim objects, or simply set to some value less than the saturation rate of $10^{6}$ per second for brighter objects, for which the count rate would need to be controlled in practice by the use of neutral density filters, or some other means. The duration of an atmospheric turbulence-corrupted realization of the classical image intensity $T_{A}$ also must be established by the user. The mean number of photo-electrons per image is then $\bar{K} T_{A}$, and the classical image intensity is normalized so that it sums to this value. This array is input to a Poisson random number generator, which returns a realization of a Poisson random process which at each location in the 
image has the mean value $\bar{K} T_{A} i_{N}(x, y ; t)$, where $i_{N}(x, y ; t)$ is the classical image intensity normalized so that $\sum_{x, y} i_{N}(x, y ; t)=1$. In general, the mean count rate per pixel is much less than unity in most cases of practical interest,

so that the most likely outcomes of the call to the Poisson random number generator at any pixel is that either one photoelectron occurred, or there were no photo-electrons. The photo-events generated in this manner are vectorized, their order is randomized, and the time step between them is presently set to be equal to $T_{A} / K$, where $K$ is the actual number of photo-electrons in the image. This last step of setting the time between photo-electron arrivals to be equal is an approximation to the real physical process. However, the images simulated in this manner form a spatial Poisson random process when the photo-events are accumulated into images for post processing.

The temporal resolution for the 3-D images can be established by considering the size of the arrays holding the 3-D object model, and the number of slabs of the object it is feasible to hold in memory. Our experience with the models we have used thus far is that setting the temporal resolution of the RULLI at on the order of $0.33 \mathrm{~ns}$ yields $5 \mathrm{~cm}$ range resolution, providing a reasonable tradeoff between the range resolution and memory requirements of the simulation. The mean count rate can, at least in principal, be controlled by controlling the average power of the illumination laser while accounting properly for the range and reflectivity of the target.

The comparative SNR provided by a RULLI detector and a conventional CCD detector is now discussed. We will consider light falling on a single CCD detector-sized patch of a RULLI detector, and a single detector of a CCD. One physical scenario under which this is a reasonable model is Nyquist sampling in the image plane for both the RULLI detector and the CCD detector. Let the mean number of photons per second falling on this region of each detector be represented by $\bar{P}$, which would be determined by dividing the optical power falling on this detector patch by the energy in a photon. The mean rate of photo-electron generation in the RULLI detector is then $\eta_{R} \bar{P}$, where $\eta_{R}$ is the quantum efficiency of the RULLI detector, and the mean rate of photo-electron generation in the CCD detector is $\eta_{C} \bar{P}$, where $\eta_{C}$ is the quantum efficiency of the CCD detector. Since it is necessary to integrate for a finite amount of time to form images, we establish an integration time $\tau$ which is assumed to be the same for both the RULLI detector and the CCD. The mean number of photo-electrons per integration time for the RULLI detector is thus $\bar{K}_{R}=\tau \eta_{R} \bar{P}$, and the mean number of photo-electrons per integration time in the CCD detector is $\bar{K}_{C}=\tau \eta_{C} \bar{P}$ The SNR of the RULLI detector output is then [Roggemann, 1996] $S N R_{R}=\sqrt{\bar{K}_{R}}=\sqrt{\tau \eta_{R} \bar{P}}$, and the SNR of the CCD detector output is $S N R_{C}=\bar{K}_{C} / \sqrt{\bar{K}_{C}+\sigma_{R N}^{2}}={ }^{\tau \eta_{C}} \bar{P} / \sqrt{\tau \eta_{C} \bar{P}+\sigma_{R N}^{2}}$, where $\sigma_{R N}^{2}$ is the variance of the read noise associated with the CCD readout. The ratio of these SNRs is a reasonable comparative metric:

$$
\frac{S N R_{R}}{S N R_{C}}=\left(\frac{1}{\tau \eta_{C} \bar{P}}\right) \sqrt{\tau \eta_{R} \bar{P}\left(\tau \eta_{C} \bar{P}+\sigma_{R N}^{2}\right)}=\sqrt{\frac{\eta_{R}}{\eta_{C}}+\frac{\eta_{R} \sigma_{\text {RN }}^{2}}{\tau \eta_{C}^{2} \bar{P}}}
$$

When this ratio of SNRs exceeds unity, the RULLI detector provides superior SNR performance, and conversely. Analysis of this result shows that for reasonable values of the device parameters, the SNR performance of the RULLI detector exceeds the performance of a CCD at very low count rates. We now describe the 2-D imaging simulation in more detail.

\section{2-D Simulation Description}

A previously existing simulation of imaging through atmospheric turbulence [Roggemann, 1996] was integrated with the RULLI detector model to create a time series of speckled images which were used to create a time series of photoelectron locations and arrival times. Since a RULLI detector has no fixed integration time, it is possible to bin the photoelectrons into user-selected integration times to optimize the tradeoffs between exposure time, light level, and wind speed [Welsh, 1995]. The simulation used here has a pupil model with a $3.63 \mathrm{~m}$ diameter primary mirror, and a $0.18 \mathrm{~m}$ diameter secondary mirror embedded in a $256 \times 256$ sample array. The sample spacing was $\Delta x=2.84 \mathrm{~cm}$, so that the pupil diameter spans one half of the array size, and the resulting images created by the simulation are Nyquist sampled at 
an angular sample spacing of $73.89 \mathrm{nRad}$ [Goodman, 1996]. The effects of atmospheric turbulence were simulated by placing a single phase screen with user specified Fried parameter $r_{0}$ in the telescope pupil. The phase screens were created using a widely available technique [Brennan, 2006]. The phase screens were created in $2000 \times 2000$ sample arrays so that the effects of uniform wind could be modeled by moving the phase screen during the course of the data collection simulation. The object used in the simulation was the widely used OCNR satellite rendering placed at a range of $500 \mathrm{~km}$ from the aperture, and scaled to span approximately $10 \mu \mathrm{Rad}$. The mean wavelength of the simulation was $550 \mathrm{~nm}$, and $r_{0}=15 \mathrm{~cm}$ at $500 \mathrm{~nm}$. The wind speed was set at $20 \mathrm{~m} / \mathrm{s}$, and the time step for moving the phase screens was set at $250 \mu$ s, which is thus also the lifetime of any realization of the classical intensity falling on the RULLI detector. The Greenwood frequency for this arrangement is $71 \mathrm{~Hz}$. The phase screens were shifted using a linear interpolation technique. Two cases of mean photo-electron generation rate were run: $\bar{K}=10^{6}$ per second, and $\bar{K}=750,000$ per second. The simulation was run to collect a total of $4 \mathrm{~s}$ of data.

In addition to demonstrating the imaging performance of the RULLI detector for an extended object, we also desire to provide a comparison of RULLI and CCD imaging performance for the same object. To accomplish this we define the CCD parameters in terms of the RULLI parameters, and then establish the mean count rate for the CCD detector. This can be accomplished by assuming quantum efficiencies for both detectors, and additionally setting a read noise level for the CCD detector. We assumed that the quantum efficiency of the RULLI detector at $500 \mathrm{~nm}$ is $5 \%$, and the quantum efficiency of the CCD detector was $80 \%$ at this wavelength. Hence, the mean photo-electron generation rate in the CCD is $\bar{K}=16 \times 10^{6}$ per second. The integration time of the CCD detector was set at $20 \mathrm{~ms}$, and the RMS read noise level was set at 20 electrons per readout per pixel. The simulation was run both for the object, and a reference star so that speckle imaging techniques could be used to reconstruct an estimate of the object. The images were post-processed using the bispectrum image reconstruction technique [Roggemann, 1996] for RULLI images that were also accumulated from $20 \mathrm{~s}$ of data. The resulting images are presented in Fig. 1. For comparison purposes, we repeated this simulation for mean count rates in the RULLI detector of $750,000 \mathrm{~s}^{-1}$, and $500,000 \mathrm{~s}^{-1}$, and these results are shown in Figs. 2 and 3, respectively. Inspection of Figs. 1, 2, and 3 shows that in this light level regime the quality of the images obtained from the RULLI data remains reasonably constant, while the quality of the images obtained from the CCD camera steadily degrades due to the presence of read noise in the measurements.

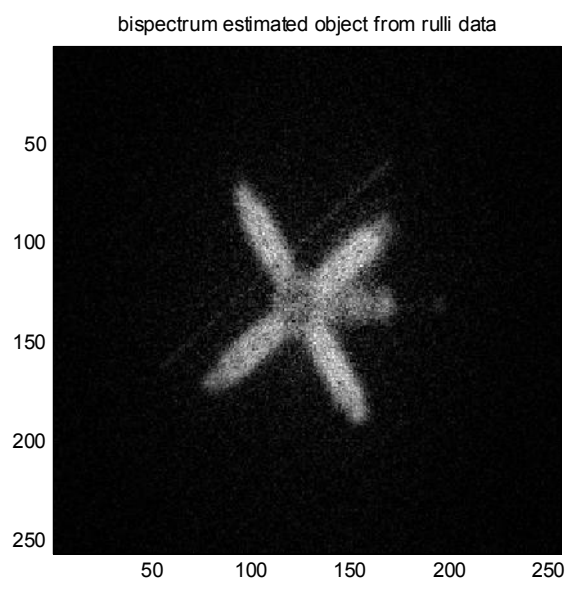

(a)

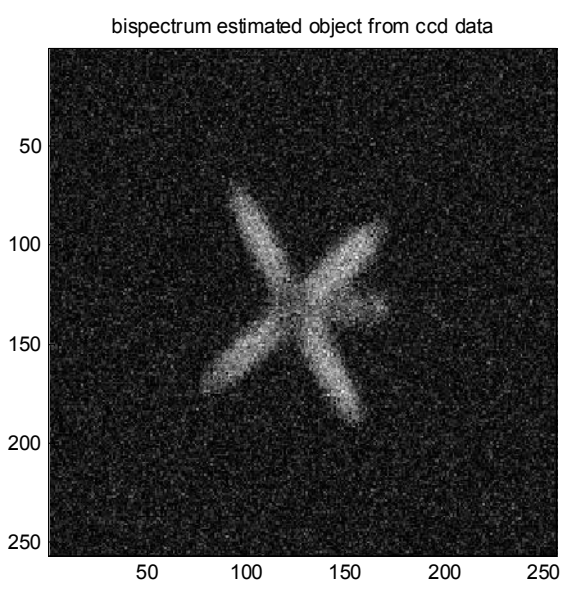

(b)

Figure 1. Example bispectrum reconstructions of the OCNR satellite for the case of the RULLI sensor operating at a mean photo-electron generation rate of $10^{6} \mathrm{~s}^{-1}$ : (a) reconstruction from RULLI data using $20 \mathrm{~ms}$ integration times, and (b) reconstruction from CCD data. 


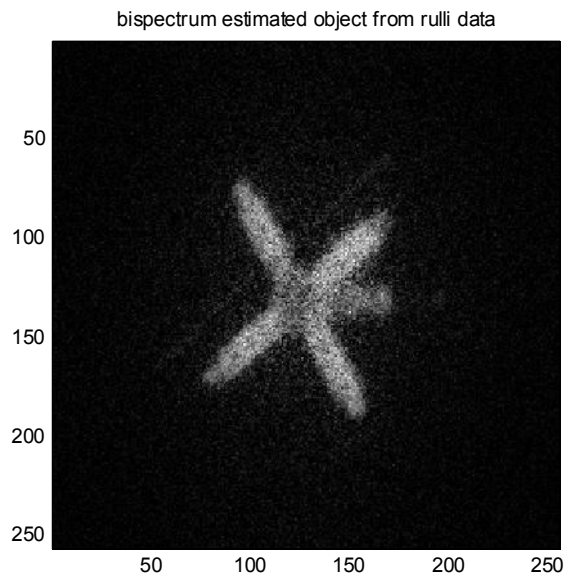

(a)

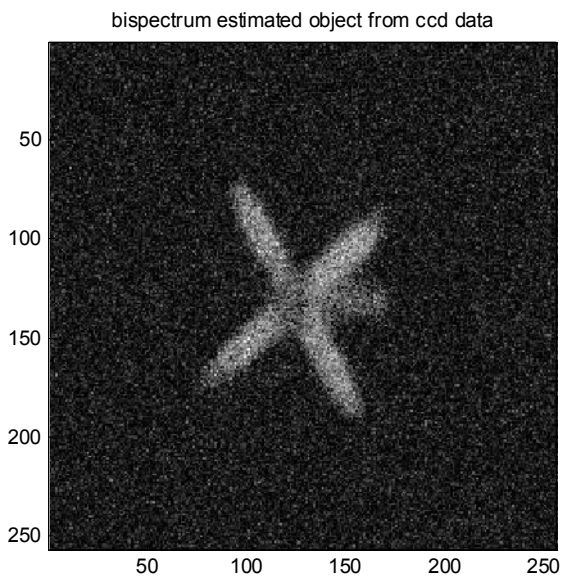

(b)

Figure 2. Example bispectrum reconstructions of the OCNR satellite for the case of the RULLI sensor operating at a mean photo-electron generation rate of $750,000 \mathrm{~s}^{-1}$ : (a) reconstruction from RULLI data using 20 ms integration times, and (b) reconstruction from CCD data.

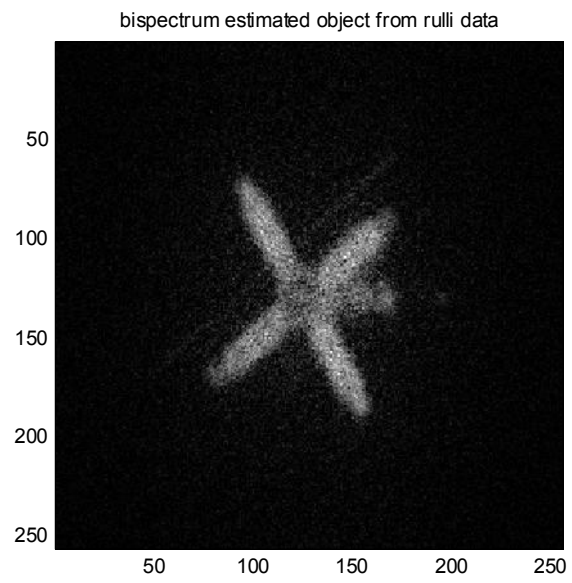

(a)

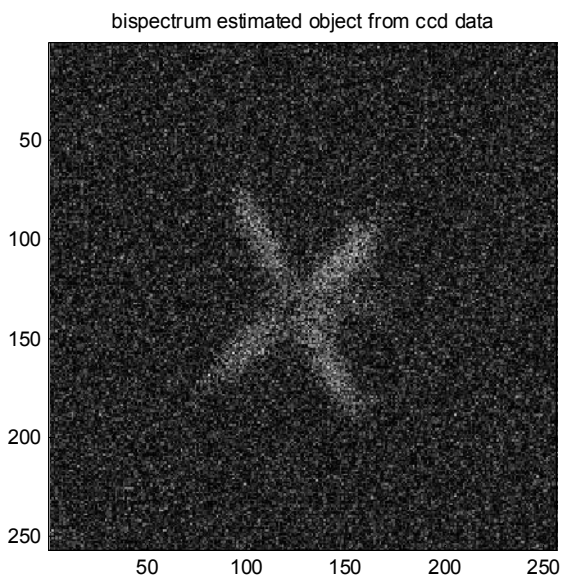

(C)

Figure 3. Example bispectrum reconstructions of the OCNR satellite for the case of the RULLI sensor operating at a mean photo-electron generation rate of $500,000 \mathrm{~s}^{-1}$ : (a) reconstruction from RULLI data using 20 ms integration times, and (b) reconstruction from CCD data.

\section{3-D Simulation Description}

The 3-D imaging simulation is based on illumination of the target with a very short pulse laser of wavelength $532 \mathrm{~nm}$. It was assumed that the launch time of each pulse is known. We now present a discussion of the illumination, scattered, and received fields.

The illuminating laser beam is modeled as a continuous train of short $(<<100 \mathrm{ps})$ pulses at a repetition rate of $1 \mathrm{MHz}$ and a wavelength of $532 \mathrm{~nm}$. The pulse duration of the laser is $T_{P}=8 \mathrm{ps}$, and the repetition rate is on the order of $1 \mathrm{MHz}$. This beam is passed through a $D=60 \mathrm{~cm}$ diameter aperture for the uplink. The $\mathrm{e}^{-1}$ radius of the field strength which passes $95 \%$ of the power of a Gaussian $\mathrm{TEM}_{00}$ beam is $w_{0}=\frac{1}{1.224}\left(\frac{D}{2}\right)=24.5 \mathrm{~cm}$. Since diffraction and turbulence effects will not be sufficient to broaden the beam enough to fully illuminate the target, it is necessary to diverge the 
outgoing beam at the pupil. This is accomplished with a negative lens placed in the pupil of the transmitter. The relationship between the divergence angle $\theta_{D}$ and the focal length of this negative lens $f_{l}$ is $f_{l}=-\left(\frac{D}{2}\right)\left(\frac{1}{\theta_{D}}\right)$, and in the present case $\theta_{\mathrm{D}}=10 \mu \mathrm{Rad}$. The expression for the electric field due to the beam leaving the aperture is thus

$$
E(x, y, z=0)=E_{0} \exp \left[-\left(x^{2}+y^{2}\right) w_{0}^{2}\right] \exp \left[-j \frac{k}{2 f_{l}}\left(x^{2}+y^{2}\right)\right],
$$

where $E_{0}$ is the peak strength of the field, and $k=2 \pi / \lambda$.

While the free space propagation characteristics of Gaussian beams are well understood [Verdeyen, 1995; Ishimaru, 1978], atmospheric turbulence-induced errors cause detrimental effects on the propagating beam [Beland, 1993]. These effects include: (1) broadening - the transmitted beam is broadened more than would be predicted by diffraction in free space alone, distributing the energy over a wider area; (2) wandering - the beam appears to "wander" in the sense that the centroid of the intensity pattern at the target or receiver plane will move randomly; and (3) speckling - on short time scales the intensity distribution will be "speckled", exhibiting high spatial frequency modulation in the intensity pattern. The long time average $\mathrm{e}^{-1}$ radius of the intensity pattern $\left\langle\rho_{L}^{2}\right\rangle$ after propagation through a turbulent path of length $L$ has been analyzed, with the result [Beland, 1993]

$$
\left\langle\rho_{L}^{2}\right\rangle=w^{2}(z)+\frac{4 L^{2}}{\left(k \rho_{0}\right)^{2}}
$$

where,

$$
\rho_{0}=\left[1.46 k^{2}\left(\sec \theta_{z}\right) \int_{0}^{L} C_{n}^{2}(\eta)(1-\eta / L)^{5 / 3} \mathrm{~d} \eta\right]^{-3 / 5}
$$

where $\theta_{z}$ is the zenith angle, and $C_{n}^{2}(\eta)$ is the structure constant of the turbulence as a function of altitude, $\eta$, and $w(z)$ is the radius of the beam waist a distance $z$ from the aperture [Verdeyen, 1995; Ishimaru, 1978]. In the present case of viewing at $\theta_{z}=0 \mathrm{deg}$, through the Maui3 turbulence profile which has $L=20 \mathrm{~km}$ yields $\rho_{0}=8.1 \mathrm{~cm}$. As an example, at the top of the atmosphere, the first term in the expression for $\left\langle\rho_{L}^{2}\right\rangle$, which is due to diffraction effects, evaluates to $w^{2}(z)=0.0602$, and the second term in the expression for $\left\langle\rho_{L}^{2}\right\rangle$, which is due to turbulence induced broadening, evaluates to $\frac{4 L^{2}}{\left(k \rho_{0}\right)^{2}}=0.0016$, which yields a theoretical value for the $\mathrm{e}^{-1}$ radius of the intensity pattern of $24.9 \mathrm{~cm}$. We have tested this simulation extensively, and found that it matches theory well [Sergeyev, 2008]. Using this simulation we find that the $\mathrm{e}^{-1}$ radius of the average intensity pattern due to the illuminating laser is approximately $20 \mathrm{~m}$.

In a pulsed laser illumination system the length of the pulse $c T_{p}$, where $c$ is the speed of light. In practice it is often the case that $c T_{P} \ll L_{T}$, where $L_{T}$ is the length of the target along the direction of propagation of the laser. As a result, the duration of the returned pulse is generally much longer than the duration of the transmitted pulse. This is due to the fact that as the leading edge of the transmitted pulse falls on the surface of the target closest to the transmitter light is immediately scattered back in the direction of the receiver, and this process continues until the trailing edge of the pulse has passed by the target.

We now discuss the down welling beam. The model developed under this program is for a Lambertian scattering target. A Lambertian target is "optically rough", in the sense that complex field reflectivity of the scattering surface contains a random phase term, and this random phase is uniformly distributed on $(-\pi, \pi)$ [Goodman, 2006]. In addition, the projected area of each scattering surface in the direction of the receiver is foreshortened by a factor of $\cos \phi$, where $\phi$ is 
the angle between a line connecting the target and the receiver, and the local surface normal of the object. In calculations of radiometric power transfer in optical systems it is necessary to account for this foreshortening of the area of the target by including a multiplicative factor of $\cos \phi$ in the calculations of the projected area for power transfer calculations, and a factor of $\sqrt{\cos \phi}$ in calculations involving field quantities.

A wave optics propagator [Roggemann, 1998] computes the classical field falling on the collecting aperture. This field is converted to a simulated RULLI data stream in the following manner. The classical intensity image associated with the field intercepted by the aperture is computed using standard Fourier optics techniques [Goodman, 1996] for each time slice. The temporal resolution of the receiver model for the results presented below is $0.33 \mathrm{~ns}$, yielding a range resolution of approximately $5 \mathrm{~cm}$ for the system. A 3-D rendering of the ARGOS satellite was obtained, and sliced along the line of sight between the sensor and the satellite into layers $5 \mathrm{~cm}$ thick. A series of laser pulses were propagated through the atmosphere, from the top of the atmosphere to the target plane, and used to illuminate the satellite. The intensity illuminating the satellite was spatially varying. The life time of the atmospheric turbulence was set at $10 \mathrm{~ms}$, so that every $10 \mathrm{~ms}$ a new realization of the 3-D volume of the atmosphere was obtained. The laser was run at a pulse rate of 100,000 pulses per second, and the mean count rate at the receiver was set at 5 photo-electrons per pulse. Data was simulated for a time interval of $0.5 \mathrm{~s}$, so that the mean number of photo-electrons detected during this time is 250,000 . The images were also sampled at the Nyquist rate of $73.89 \mathrm{nRad}$. The range of the target was $9.76 \times 10^{5} \mathrm{~m}$. The RULLI data was assembled into both a 2-D image and a 3-D object rendering using the approach described above, and the results are shown in Fig. 4. Inspection of Fig. 4 shows that that excellent 2-D and 3-D renderings of objects can be created from RULLI measurements.

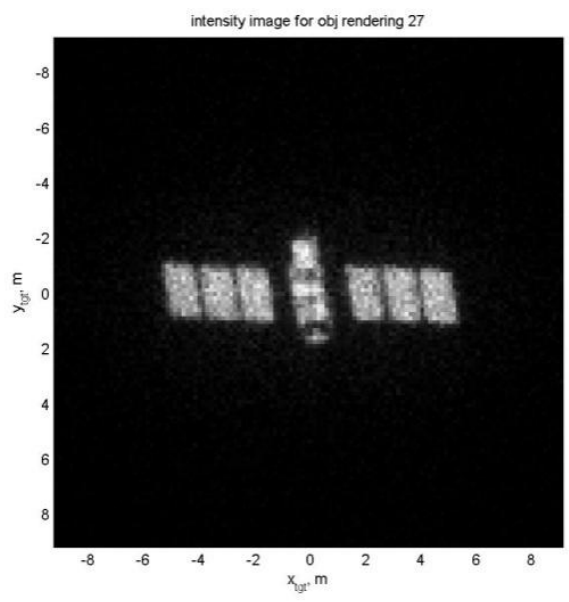

(a)

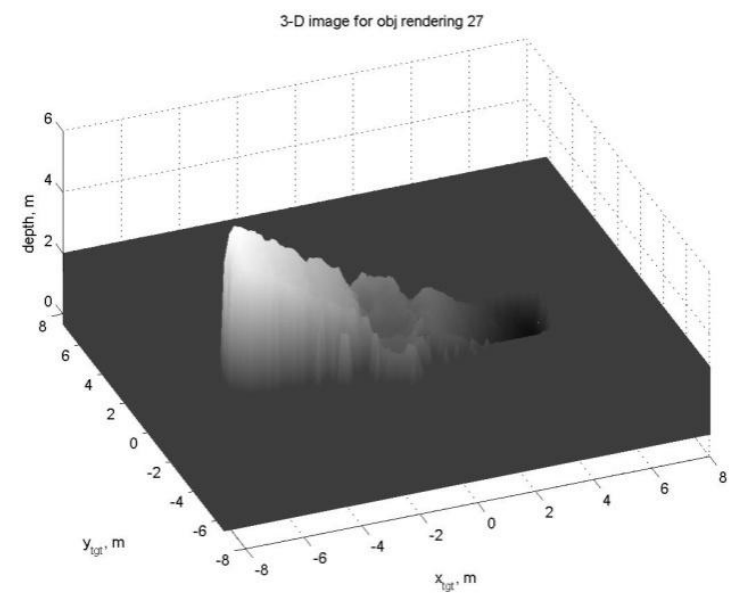

(b)

Figure 4. (a) 2-D image created from the RULLI data; (b) 3-D object rendering obtained from the simulated RULLI data

\section{Conclusion}

The photon-limited nature of the detection process in RULLI detectors and the high temporal resolution which can be obtained from a RULLI detector afford unique opportunities for making measurements of space objects. The results presented here show the promise of SSA systems based on RULLI detectors. However, much work remains to be done, including experimental work to demonstrate in actual practice the promise shown in the modeling and simulation efforts conducted so far.

\section{$\underline{\text { Acknowledgement }}$}

This work was sponsored by the Air Force Research Laboratory, contract number FA9451-07-D-0203. 


\section{$\underline{\text { References }}$}

[Beland, 1993] R. R. Beland, "Propagation through atmospheric turbulence", Chapter 2, Volume 2, IR/EO Handbook, SPIE Optical Engineering Press, Bellingham, WA, 1993.

[Brennan, 2006] Terry J. Brennan and Phillip H. Roberts, "AOTools - The Adaptive Optics Toolbox for use with MATLAB", the Optical Sciences Company, Anaheim, CA, 2006. (available on-line at: http://www.tosc.com/downloads/AOToolsUG.pdf)

[Goodman, 1996] J. W. Goodman, Introduction to Fourier Optics, $3^{\text {rd }}$ Edition, McGraw Hill Company, Inc., New York, 1996.

[Ho, 1999] Cheng Ho, Kevin L. Albright, Alan W. Bird, Jeffrey Bradley, Donald E. Casperson, Miles Hindman, William C. Priedhorsky, W. Robert Scarlett, R. Clayton Smith, James Theiler, and S. Kerry Wilson, "Demonstration of literal three-dimensional imaging", Appl. Opt., vol. 38, p1833-1840, 1999.

[Ishimaru 1978] A. Ishimaru, "The beam wave case and remote sensing", in Laser Beam Propagation in the Atmosphere, Ed. J. W. Strohbehn, Vol. 25, Topics in Applied Physics, Springer-Verlag, Berlin, 1978.

[Priedhorsky, 1996a] William C. Priedhorsky, R. Clayton Smith, and Cheng Ho, "Laser ranging and mapping with a photon-counting detector", Appl. Opt., vol. 35, p441-452, 1996.

[Priedhorsky, 1996b] William C. Priedhorsky, "Contrast and signal-to-noise ratio in long-distance starlight imaging", Appl. Opt., vol. 35, p4173-4179, 1996.

[Priedhorsky, 2005] William Priedhorsky and Jeffrey J. Bloch, "Optical detection of rapidly moving objects in space", Appl. Opt., vol. 44, p423-433, 2005.

[Roggemann, 1996] Michael C. Roggemann, and B. M. Welsh, Imaging Through Turbulence, CRC Press, Boca Raton, FL, 1996.

[Roggemann, 1998] M. C. Roggemann, and T. J. Schulz, "Algorithm to increase the largest aberration which can be reconstructed from Hartmann sensor measurements”, Appl. Opt.,.vol. 37, p4321-4329, 1998.

[Sergeyev, 2008] A. V. Sergeyev, P. Piatrou, and M. C. Roggemann, "Bootstrap beacon creation for overcoming the effects of beacon anisoplanatism in a laser beam projection system", Appl. Opt., vol. 47, p2399-2413, 2008.

[Verdeyen, 1995] J. T. Verdeyen, Laser Electronics, $3^{\text {rd }}$ Edition, Prentice-Hall, Upper Saddle River, NJ, 1995.

[Welsh, 1995] B. M. Welsh, "Speckle imaging signal-to-noise ratio performance as a function of frame integration time", J. Opt. Soc. Am. A, vol. 12, p1364-1374, 1995. 